ARTÍCULO

\title{
El Convenio de Estambul en Francia y en España: tareas pendientes ${ }^{\bullet}$
}

\section{The Istanbul Convention in France and Spain: Unresolved Matters}

\author{
Alicia Ginebra Brox Sáenz de la Calzada \\ Investigadora predoctoral F.P.U (Ministerio de Ciencia, Innovación y Universidades) \\ Laboratorio de Sociología Jurídica \\ Facultad de Derecho \\ Universidad de Zaragoza
}

Fecha de recepción 04/03/2020 | De aceptación: 15/10/2020 | De publicación: 28/12/2020

\section{RESUMEN.}

En 2020 se cumplieron seis años desde la ratificación del Convenio de Estambul (2011) por parte de España y Francia. En su momento, el texto fue ampliamente aplaudido porque supuso un hito en Europa en la lucha contra la violencia ejercida contra la mujer. No obstante, pasado el entusiasmo inicial que despertó en algunos sectores, conviene hacer ahora un balance de sus logros, así como de aquellas medidas que, por diversos motivos, siguen en ciernes, o se han quedado directamente en el tintero.

\section{PALABRAS CLAVE.}

Convenio de Estambul, violencia de género, perspectiva de género, Francia, España

\section{ABSTRACT.}

Six years after its ratification by France and Spain, the Istanbul Convention (2011) is still one of the most powerful European tools on fighting violence against women. Nonetheless, after the initial enthusiasm that aroused in some sectors, it suits now to do a balance of all the achievements that have finally been carried out, as well as those other measures that, for various reasons, are budding or, directly, haven't been taken.

\section{KEY WORDS.}

Istanbul Convention, Gender-based Violence, Gender Perspective, France, Spain

\footnotetext{
- Proyectos competitivos en cuyo marco se ha desarrollado el trabajo: proyecto Grupo de Referencia Laboratorio de Sociología Jurídica (09_17R), del Gobierno de Aragón; El Tratamiento de la Violencia de Género en la Administración de Justicia. Implementación y eficacia de la LO 1/2004 (DER2014-55400-R).
} 
Sumario: 1.Introducción. 2. Las mejoras: un impulso para el desarrollo de la perspectiva de género en Derecho penal 2.1. La ampliación del concepto de violencia contra las mujeres 2.2. El desarrollo penal de la regulación contra dicha violencia 2.3. La configuración generoespecífica de ciertas infracciones 3. Las carencias: la vaguedad conceptual, un posible freno a la capacidad transformadora del texto 3.1. La "perspectiva de género», una herramienta no definida 3.2. La opacidad del concepto de violencia doméstica 3.3. La trata con fines de explotación sexual, una violencia olvidada 3.4. El consentimiento sexual: un concepto central indeterminado 4. Conclusión 5. Referencias bibliográficas

En recuerdo de Manuel Calvo García ${ }^{1}$

\section{Introducción}

El pasado 19 de noviembre de 2019, el Grupo de Expertas en Acción contra la Violencia sobre la Mujer y la Violencia Doméstica (en adelante, GREVIO) publicaba el Informe sobre el grado de implementación ${ }^{2}$ logrado por Francia en relación con el Convenio del Consejo de Europa sobre prevención y lucha contra la violencia contra la mujer y la violencia doméstica, conocido como Convenio de Estambul (en adelante, CE). En él saludaba la «gran determinación» con la que las autoridades francesas habían llevado a cabo las reformas legislativas, pero subrayaba también las lagunas existentes en el ordenamiento jurídico. El Informe señalaba el progresivo compromiso político que las instituciones francesas habían asumido en la lucha contra esta lacra, desde el primer plan interministerial adoptado en $2005^{3}$, hasta 2019, año en el que la cuestión se ha convertido en una prioridad política ${ }^{4}$. Sin embargo, a pesar de las incuestionables iniciativas adoptadas, lo cierto es que paliar este tipo de violencia sigue siendo una tarea pendiente en el país galo. Sin ir más lejos, las cifras de feminicidios cometidos en el ámbito sentimental de estos tres últimos años resultan sumamente preocupantes. En 2016, 123 mujeres

\footnotetext{
${ }^{1}$ Catedrático de Filosofía del derecho y Sociología jurídica de la Universidad de Zaragoza, fallecido el día 21 de junio de 2020.

${ }^{2}$ Informe de evaluación (de referencia) del grado de implementación del Convenio de Estambul (Francia), Rapport sur les mesures d'ordre législatif et autres donnant effet aux dispositions de la Convention du Conseil de l'Europe sur la prévention et la lutte contre la violence à l'égard des femmes et la violence domestique (France), GREVIO/Inf(2019)16, Grupo de Expertos en la lucha contra la violencia contra la mujer y la violencia doméstica (GREVIO), Consejo de Europa, Francia, 19 de noviembre de 2019. El documento completo puede ser consultado a través del siguiente enlace: https://rm.coe.int/grevio-inf-2019-16/168098c619 [15/10/2020].

${ }^{3}$ Fuente: https://www.senat.fr/rap/r15-425/r15-4251.html [15/10/2020].

${ }^{4}$ El 3 de septiembre de 2019 el Estado francés declaró su intención de acabar con la violencia conyugal, abriendo para ello un periodo de tres meses de reflexión política, llamado «Grenelle des violences conjugales», durante el que el antiguo Primer Ministro Edouard Philippe y la ex Secretaria de Estado encargada de la igualdad entre hombres y mujeres, Marlène Schiappa, condujeron una serie de consultas a expertos, operadores jurídicos y parajurídicos en materia de violencia conyugal, con la finalidad de discutir sobre los recursos necesarios para acabar con esta lacra. Al término de esos tres meses, el Estado francés planteó diversas medidas políticas, disponibles a través del siguiente enlace: https://www.egalite-femmes-hommes.gouv.fr/conclusion-du-grenelles-des-violences-conjugales-de-la-mobilisation-alaction/ [15/10/2020].
} 
fueron mortalmente agredidas por sus parejas, actuales o pretéritas ${ }^{5}$; en 2017, la cifra se elevó a $130^{6}$, y en 2018 , fueron $121^{7}$.

Para progresar en la lucha contra el problema, el citado Informe elaborado por el GREVIO insta a seguir adoptando medidas que implementen las disposiciones del CE. Entre ellas, sugiere, por un lado, que se reconfigure penalmente el delito de violación, que, como precisa el texto internacional, debe estructurarse en torno al «libre consentimiento de la víctima», con independencia del comportamiento del agresor. En Francia, la redacción actual del delito conlleva analizar la falta de consentimiento a la luz de la conducta del agresor ${ }^{8}$.

Por otro lado, el Informe también constata la «coexistencia de diversos conceptos de violencia contra la mujer», alentando a su homogeneización para una mejor comprensión del fenómeno. En efecto, a diferencia de nuestro sistema, en el cual, a raíz de la Ley Orgánica 1/2004 de Medidas de Protección Integral contra la Violencia de Género, se ha extendido el uso de la expresión «violencia de género»" en el país galo, incluso en el ámbito parajurídico, se alude a este problema con diversos términos, como son «violencia familiar», «violencia específicamente ejercida contra las mujeres», «violencia en la pareja», «violencias ejercidas contra las mujeres», «violencia de género», «violencia sexoespecífica», o «violencias sexistas y sexuales» ${ }^{10}$. Al no existir una categoría homogénea que englobe las diversas formas de dicha violencia, coexiste una multiplicidad terminológica que, lejos de fomentar una comprensión global, resulta poco diáfana y genera dudas en torno a las raíces del problema ${ }^{11}$.

\footnotetext{
${ }^{5}$ Ver el documento «Principales datos sobre la Encuesta sobre las muertes violentas en el ámbito de la pareja», año 2016, Delegación para

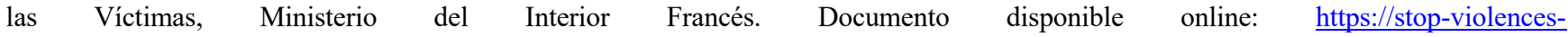
femmes.gouv.fr/IMG/pdf/enquete sur les morts violentes au sein du couple 2016 - principaux enseignements - miprof.pdf [15/10/2020].

${ }^{6}$ Ver el documento «Estudio nacional sobre las muertes violentas en el ámbito de la pareja», año 2017, Delegación para las Víctimas, Ministerio del Interior Francés. Disponible online: https://www.egalite-femmes-hommes.gouv.fr/wp-content/uploads/2018/11/Etudenationale-sur-les-morts-violentes-au-sein-du-couple-annee-2017.pdf [15/10/2020].

${ }^{7}$ Ver el documento «Estudio nacional sobre las muertes violentas en el ámbito de la pareja», año 2018, Delegación para las Víctimas, Ministerio del Interior Francés. Disponible online: https://www.interieur.gouv.fr/Actualites/Communiques/Etude-nationale-relative-auxmorts-violentes-au-sein-du-couple [15/10/2020].

${ }^{8}$ Art. 222-23 del Código Penal francés.

${ }^{9}$ Ello, a pesar de que la citada Ley Orgánica 1/2004 hace un uso limitado de la expresión «violencia de género», en la medida en que circunscribe su ámbito de aplicación a aquellos actos que provienen de la pareja, ya sea actual o pretérita.

${ }^{10} \mathrm{~A}$ todas estas denominaciones se refiere el citado Informe de evaluación (de referencia) del grado de implementación del Convenio de Estambul (Francia), ob. cit., p. 12. El documento completo puede ser consultado a través del siguiente enlace: https://rm.coe.int/grevio-inf2019-16/168098c619 [15/10/2020].

${ }^{11}$ En este sentido, ver VENTURA FRANCH, A., «El CE y los sujetos de la violencia de género. El cuestionamiento de la violencia doméstica como categoría jurídica», UNED. Revista de Derecho Político, n. 97, 2016, p. 182.
} 
En 2020 se cumplían seis años desde la ratificación del Convenio de Estambul por parte de Francia y España. En su momento, el texto fue ampliamente aplaudido por la doctrina porque supuso un hito en Europa. No obstante, pasado el entusiasmo inicial, y, al hilo del Informe citado, conviene hacer ahora un balance de sus logros y de aquellas otras medidas que, por diversos motivos que veremos más adelante, se han quedado en el tintero. Con esa finalidad, este artículo aborda en primer lugar las novedades más destacables que el texto del CE planteó en su momento, en concreto, en lo relativo a la implementación de la perspectiva de género en las herramientas jurídicas de lucha contra la violencia contra las mujeres. En segundo lugar, se hará un análisis sobre los mecanismos internos del CE que limitan su potencial transformador.

\section{Las mejoras introducidas por el $\mathrm{CE}$ : un impulso para el desarrollo de la perspectiva de género} en Derecho penal

Elaborado en 2011 y ratificado por Francia y por España en $2014^{12}$, el CE ha supuesto varios logros en la lucha contra la violencia de género. Ambicioso en su contenido, se trata del primer instrumento jurídico europeo en abordar específicamente la cuestión de la violencia de género como una violación de derechos humanos, lo que, al menos simbólicamente, implica asumir «un lenguaje común que refleja valores universales y que posee un sistema jurídico ampliamente compartido que articula estándares básicos de una vida digna» ${ }^{13}$. Por otro lado, el CE es jurídicamente vinculante, lo cual supone que, a diferencia de los otros instrumentos internacionales de soft law sobre violencia de género, impele a los Estados signatarios a adoptar las medidas necesarias para implementarlo. Entre ellas, figura la obligación de sancionar jurídicamente todas las formas de violencia previstas en el texto. Ello supone

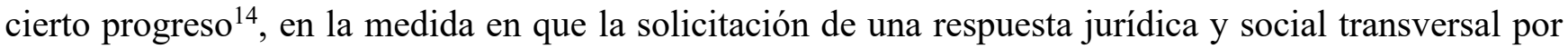

\footnotetext{
${ }^{12}$ España ratificó dicho Convenio el 10 de abril de 2014, mientras que Francia lo hizo el 4 de julio de ese mismo año.

${ }^{13}$ Ver EDWARDS, A., Violence against women under International Human Rights Law, Cambridge University Press, 2011, pág. 11. Ver también FRIEDMAN, E., J, «Women's human rights: the emergence of a movement perspective», en PETERS, J., y WOLPER, A., (eds.), Women's Rights, Human Rights: International Feminist Perspectives, Nueva York, Routledge, 1995; GARCÍA INDA, A., y LOMBRADO, E., (coords.), Género y Derechos Humanos, Terceras Jornadas Derecho Humanos y libertades fundamentales, Huesca, Mira Ed., 2002; CALVO GARCÍA, M., «La violencia de género como violación de Derechos Humanos. El papel de los movimientos sociales en la lucha por los derechos», en A.A.V.V., Historia de los Derechos Fundamentales, Tomo IV, Siglo XX, Madrid, Dykinson, 2014; y GIL RUIZ, J. M. (Ed.), El Convenio de Estambul como marco de derecho antisubordiscriminatorio, Madrid, Dykinson S.L., 2018, entre otros.

${ }^{14}$ Ver ALIJA FERNÁNDEZ, R. A., «La violencia doméstica contra las mujeres y el desarrollo de estándares normativos de derechos humanos en el marco del Consejo de Europa», Revista General de Derecho Europeo 24 (2011), p. 3: «En el ámbito regional europeo, el Consejo de Europa ha mostrado en la última década una particular sensibilidad frente a la violencia contra las mujeres, y en particular frente a la violencia doméstica, dado el carácter paneuropeo del fenómeno y el compromiso de la organización de salvaguardar los derechos humanos. No obstante, la reacción frente a esta lacra ha sido más bien tardía, en comparación con otros sistemas regionales, sobre todo si se toma como referencia la fecha de adopción de estándares jurídicos obligatorios en materia de violencia contra la mujer. De hecho, se puede observar que ha habido una cierta resistencia a imponer obligaciones jurídicas internacionales a los Estados en relación con las medidas a adoptar para enfrentar el problema de la violencia contra las mujeres desde la perspectiva de género».
} 
parte de los Estados, implicando el reconocimiento a nivel interno del carácter poliédrico de la violencia contra las mujeres por motivos de género, conlleva la indicación de que los ordenamientos jurídicos se replanteen el concepto que tenían sobre dicha violencia a la luz de la evolución seguida por la representación social del problema ${ }^{15}$.

En este sentido, el tratado opera una apertura conceptual que se articula en tres estadios, consistentes, por un lado, en la obligación de ampliar a nivel interno el concepto de violencia contra las mujeres; por otro, en la recomendación de regular algunas de esas manifestaciones violentas mediante el Derecho penal; y, por último, en la conveniencia que plantea a los Estados para que introduzcan en sus legislaciones algunos de esos delitos con una configuración generoespecífica ${ }^{16}$. A continuación, explicaremos cada una de estas tres etapas.

\subsection{La ampliación del concepto de violencia contra las mujeres}

En primer lugar, haciéndose eco de Resoluciones y Declaraciones internacionales previas, el CE aborda la violencia contra las mujeres de forma muy extensa. Mediante su ratificación, los Estados signatarios aceptan reconocer tanto la existencia como la amplitud del problema. Ello se deriva del art. 3 a) del texto, que precisa que la violencia contra las mujeres [sic] es una violación de los derechos humanos y una forma de discriminación contra las mujeres, y designa todos los actos de violencia basados en el género ${ }^{17}$. Ese mismo artículo también define la violencia contra las mujeres por razones de género [sic] como «toda violencia contra una mujer porque es una mujer o que afecte a las mujeres de manera desproporcionada» ${ }^{18}$.

Conjugados ambos artículos, tanto las violencias cometidas contra las mujeres como aquellas que se infligen sobre ellas por el mero hecho de ser mujeres parecen remitir a realidades parejas, cuando no

\footnotetext{
${ }^{15}$ Ver, en este sentido, DIONISI-PEYRUSSE, A. ; PICHARD, M., «La prise en compte des violences conjugales en matière d'autorité parentale», Actualité juridique. Famille, Dalloz, 2018, pp. 34-37, disponible online: https://halshs.archives-ouvertes.fr/halshs-02263055 $[15 / 10 / 2020]$.

${ }^{16}$ Para un análisis detallado de la pertinencia de la creación de este tipo de figuras, ver LAURENZO COPELLO, P., «¿Hacen falta figuras generoespecíficas para proteger mejor a las mujeres?», Estudios Penales y Criminológicos, vol. XXXV (2015), pp. 783-830.

${ }^{17}$ Art. 3 a del CE: «por "violencia contra las mujeres" se deberá entender una violación de los derechos humanos y una forma de discriminación contra las mujeres, y designará todos los actos de violencia basados en el género que implican o pueden implicar para las mujeres daños o sufrimientos de naturaleza física, sexual, psicológica o económica, incluidas las amenazas de realizar dichos actos, la coacción o la privación arbitraria de libertad, en la vida pública o privada». Ver LOUSADA AROCHENA, J. F., «El convenio del Consejo de Europa sobre prevención y lucha contra la violencia contra las mujeres y la violencia de género», AequAlitaS 2014 (nº 35), pp. 6-15, p. 9.

${ }^{18}$ Art. 3 d del CE.
} 
difícilmente distinguibles. El concepto de violencia contra la mujer abarca los actos basados en el género, los cuales, a su vez, se cometen contra una mujer por el hecho de serlo ${ }^{19}$.

El texto del Convenio, algo redundante, supone, no obstante, una apertura considerable respecto a lo que hasta entonces se entendía por violencia de género en Derecho interno. En concreto, en España, donde el término ya había sido reconocido por nuestro sistema jurídico, incorporar la definición del CE supondría abrir el tipo penal actual de violencia de género para incluir cualquier agresión cometida sobre una mujer por el hecho de serlo, independientemente de la relación que pudiera existir entre agresor y víctima $^{20}$. En cierta medida, esta intención fue la que motivó al legislador en $2015^{21}$ a crear una nueva circunstancia agravante genérica por razones de género ${ }^{22}$, que se aplica, en principio, a pesar de haber sido redactada en términos neutros ${ }^{23}$, a mujeres que han sido víctimas de agresiones por el mero hecho de ser mujeres ${ }^{24}$.

En Francia, donde el concepto jurídico de violencia de género no existe como tal, pero donde sí disponen de varios dispositivos penales que sancionan ciertas violencias que normalmente se cometen contra las mujeres, incorporar al Derecho interno la definición que el CE ofrece de la violencia contra las mujeres por motivos de género supone todavía mayores cambios, en la medida en que, primero, es necesario acoger la expresión de «violencia de género» en Derecho, cuestión que aún no concita

\footnotetext{
${ }^{19}$ Ver el Informe explicativo del Convenio de Estambul, Rapport explicatif de la Convention du Conseil de l'Europe sur la prévention et la lutte contre la violence à l'égard des femmes et la violence domestique, CETS n. 2010, Consejo de Europa, 2011, disponible en francés (https://rm.coe.int/16800d38c9) y en inglés (https://rm.coe.int/16800d383a), punto 36, p. 7: «El párrafo 1 indica que este convenio se aplica a todas las formas de violencia ejercida contra las mujeres, inclusive la violencia doméstica. Los redactores han estimado necesario subrayar que la gran mayoría de víctimas de violencia doméstica son mujeres». Ver también el punto 44, p. 9: «la violencia basada en el género hace referencia a todo perjuicio que pueda padecer una mujer, siendo a la vez la causa y consecuencia de las relaciones de dominación desiguales percibidas entre hombres y mujeres y que conducen a la subordinación de las mujeres en las esferas públicas y privadas». Todas las referencias a dicho Informe están hechas a su versión francesa. Las traducciones son obra de la autora.

${ }^{20}$ Sobre este punto insiste en reiteradas ocasiones el reciente Informe de evaluación del grado de implementación del Convenio de Estambul en España, documento elaborado por el grupo GREVIO. Ver Grevio Baseline Evaluation Report on legislative and other mesures giving effect to the provisions of the Council of Europe Convention on Preventing and Combating Violence against Women and Domestic Violence (Spain), GREVIO/Inf(2020)19, publicado el 25 de noviembre de 2020.

${ }^{21}$ Ley Orgánica 1/2015 de 30 de marzo. Para un análisis sobre la pertinencia y el ámbito de aplicación de dicha circunstancia, ver, por ejemplo, MARÍN DE ESPINOSA CEBALLOS, E., «La agravante genérica de discriminación por razones de género (art. 22.4 CP)», Revista Electrónica de Ciencia Penal y Criminología, RECPC 20-27 (2018), disponible a través del siguiente enlace: http://criminet.ugr.es/recpc/20/recpc20-27.pdf [15/10/2020]; SEOANE MARÍN, Ma . J., OLAIZOLA NOGALES, I., «Análisis de la circunstancia agravante de discriminación por razones de género (22.4 CP)», Estudios Penales y Criminológicos, vol. XXXIX (2019), también disponible online: http://dx.doi.org/10.15304/epc.39.5880 [15/10/2020]; RUEDA MARTÍN, Mª. A., «Cometer un delito por discriminación referente al sexo de la víctima y/o por razones de género como circunstancia agravante genérica», Revista Electrónica de Ciencia Penal y Criminología, RECPC 21-04 (2019), pp. 1-37, disponible a través del siguiente enlace: http://criminet.ugr.es/recpc/21/recpc21-04.pdf [15/10/2020]; o, por fin, AGUILAR CÁRCELES, M. M., «Circunstancias agravantes genéricas», en MORILLAS CUEVA (dir.), Estudios sobre el Código Penal reformado, Madrid, 2015, pp. 58-63, entre muchas otras referencias.

${ }^{22}$ Artículo 22.4 del Código Penal español.

${ }^{23}$ Ver MARIN DE ESPINOSA CEBALLOS, E., ob. cit., págs. 14, 15 y 16.

${ }^{24}$ Ver la Sentencia del Tribunal Supremo, Sala de lo Penal, n. 565/2018, Rec 10279/2018, de 19 de noviembre de 2018.
} 
suficiente favor ${ }^{25}$. De hecho, ya en 2004, la reforma operada en nuestro sistema jurídico por la Ley Orgánica 1/2004 fue puesta en tela de juicio en Francia, porque por lo general, además de imperar cierto culturalismo en los planteamientos doctrinales en su contra, diversos sectores del mundo jurídico del país vecino entendieron que el principio de neutralidad del Derecho penal debía primar sobre la generoespecificidad. Además, como ya disponían de una circunstancia agravante de «violencia conyugal» que permitía sancionar las agresiones cometidas por un miembro de la pareja sobre el otro, dicha reforma fue vista con escepticismo. La citada circunstancia, prevista en el artículo 132-80 del Código Penal francés, sigue todavía en vigor y se aplica a un buen número de delitos ${ }^{26}$. A efectos prácticos, cubre casi todos los casos de violencia ejercida contra la mujer que en España se tratarían como casos de «violencia de género». Aún así, la entrada en vigor del CE en Francia supuso la creación de otra circunstancia agravante, la cual, desde 2017 permite sancionar más severamente las agresiones cometidas en razón del sexo de la víctima ${ }^{27}$. Con la circunstancia en cuestión, que figura en el artículo 132-77 de Código Penal francés, se reconoce por fin la motivación sexista ${ }^{28}$ de diversas agresiones en Derecho penal $^{29}$.

\subsection{El desarrollo penal de la regulación contra dicha violencia}

Además de hacer hincapié en la amplitud del concepto de violencia contra la mujer, el mandato del CE, como hemos visto, también exhorta a los Estados a tratar la gran mayoría de dichas

\footnotetext{
${ }^{25}$ Ver, en ese sentido, BROX SÁENZ DE LA CALZADA, A., «Una aproximación al concepto de género en Derecho Penal francés y español. De la polémica a su validez constitucional», Cuadernos Electrónicos de Filosofía del Derecho, n. 40, junio 2019, pp. 23-44, disponible online: https://doi.org/10.7203/CEFD.40.13751 [15/10/2020].

${ }^{26}$ En concreto, a la violencia física prevista en los arts. 222-7 a 222-16-3 del Código Penal francés (en adelante, CPF),_al acoso moral, arts. 222-33-2 a 222-33-2-2 CPF; a las agresiones sexuales, arts. 222-22 a 222-22-2 y 222-24 CPF; a las amenazas de muerte, art. 222-18-3; y, por fin, al asesinato, art. 221-4 CPF.

${ }^{27}$ Artículo 171 de la Ley francesa ${ }^{\circ} 2017-86$ de 27 de enero de 2017 relativa a la igualdad y a la ciudadanía. Sorprende que la configuración de la circunstancia sea neutra y que se encuentre ubicada en el título III de la citada Ley, título denominado «por la igualdad real». Resulta pertinente preguntarse si la justicia social puede ser ciega al género de la víctima en los casos de violencia ejercida contra la mujer por motivos de género.

${ }^{28}$ Como precisa Carol Smart, «el concepto "sexismo" implica que es posible anular la diferencia sexual como si fuera epifenoménica y no estuviera enquistada en el modo en el que comprendemos y negociamos el orden social. Para decirlo con mayor audacia, la diferencia sexual -sea que la veamos o no como una construcción- es parte de la cultura binaria del lenguaje y del significado. Si erradicar la discriminación está supeditado a erradicar la diferenciación, tendríamos que ser capaces de pensar una cultura sin género. Así, lo que parece una solución relativamente sencilla - es decir, la incorporación en el Derecho de una terminología neutra de género- en realidad enmascara un problema mucho más profundo.» SMART, C., «La teoría feminista y el discurso jurídico», en BIRGIN, H. (comp.), El derecho en el género y el género en el derecho, Buenos Aires, Biblios, 2000, pp. 31 a 71, p. 36.

${ }^{29} \mathrm{La}$ citada agravante es incompatible con la de violencia conyugal. Ver el artículo 132-77 del CPF, que precisa que el nuevo dispositivo no será aplicable cuando la sanción prevista para la infracción ya haya sido agravada, ya sea porque se haya cometido por el cónyuge, la pareja de hecho o el compañero sentimental de la víctima o bien porque la sanción ya viene agravada porque la infracción «se ha cometido contra una persona con la intención de forzarla a contraer matrimonio o a concluir una unión o en razón de su rechazo a contraer dicho matrimonio o unión».
} 
manifestaciones violentas a través del Derecho penal, lo que ya ha supuesto un aumento de este tipo de legislación, claramente sujeta a decisiones políticas ${ }^{30}$.

Los comportamientos violentos que han de ser reprimidos penalmente son múltiples: la violencia psicológica (art. 33 del CE), el acoso entendido en su sentido más amplio (art. $34 \mathrm{CE})^{31}$, la violencia sexual, incluida la violación (art. 36), los matrimonios forzosos (art. $37 \mathrm{CE}$ ), las mutilaciones genitales femeninas (art. 38), y el aborto y la esterilización forzosos (art. 39). La obligación de recurrir al Derecho penal para tratar estas infracciones no es un asunto menor. Implica hacer uso del carácter represivo de la ley, facultad unilateral del ius puniendi, cuyo potencial simbólico y político es capital por cuanto encarna y representa los valores protegidos por una sociedad determinada ${ }^{32}$. Sin embargo, por esa misma carga simbólica, conviene evitar hacer un uso abusivo, porque entraña el riesgo de favorecer la creación de una suerte de Derecho penal simbólico ${ }^{33}$.

\subsection{La introducción de un derecho penal generoespecífico}

Finalmente, la tercera fase del proceso de regulación jurídico penal que establece el CE parece sugerir que determinadas violencias sean tratadas con una configuración generoespecífica. El texto indica que, a diferencia del resto de manifestaciones violentas, los delitos de mutilación genital (femenina), aborto y esterilización forzosos, por su carácter «sexoespecífico» ${ }^{34}$, deberán buscar solamente la protección de las víctimas de sexo femenino. Con ello, el CE da un paso más en la introducción de la

\footnotetext{
${ }^{30}$ Cabe, por ejemplo, mencionar las circunstancias agravantes precitadas, así como el reciente delito de stalking (art. 172. Ter CPE), creado en España a través de la Ley Orgánica 1/2015 de 30 de marzo por la que se lleva a cabo la reforma del Código Penal, o el de acoso sexista (outrage sexiste, art. 621-1 CPF) en Francia, creado gracias a la Ley $\mathrm{n}^{\circ}$ 2018-703 del 3 de agosto de 2018, Loi renforçant la lutte contre les violences sexuelles et sexistes. La tendencia, cada vez más marcada, de recurrir al Derecho penal para tratar este problema ya fue advertida y criticada por muchos sectores doctrinales. Entre ellos, CALVO GARCÍA, M., «La violencia de género como violación de Derechos Humanos. El papel de los movimientos sociales en la lucha por los derechos», ob. cit. supra, p. 191. Ver, también, LAURENZO COPELLO, P., «La violencia de género en el derecho penal: Un ejemplo de paternalismo punitivo», en Género, violencia y Derecho, LAURENZO, P., MAQUEDA, M. L, RUBIO, A., (coords.) Valencia: Tirant lo blanch, 2008, pp. 329-361; MAQUEDA ABREU, Mª L., «¿Es la estrategia penal una solución a la violencia contra las mujeres?», InDret, 2007, n ${ }^{\circ}$ 4-07, disponible en http://www.indret.com/pdf/475 es.pdf [15/10/2020]; o por fin, DÍEZ RIPOLLÉS, J. L.; CEREZO DOMÍNGUEZ, A. I; BENÍTEZ JIMÉNEZ, M. J., La política criminal contra la violencia sobre la mujer pareja (2004-2014). Su efectividad, eficacia y eficiencia, Valencia, Tirant lo Blanch, 2018.

${ }^{31} \mathrm{El}$ artículo 78.3 del mismo CE y los puntos 181 y 186 del Informe explicativo del Convenio de Estambul, ob. cit., habilitan sin embargo a las Partes a realizar reservas sobre la naturaleza penal de la regulación, permitiéndoles regular estas manifestaciones mediante otras ramas jurídicas.

${ }^{32}$ Ver el texto de DELMAS-MARTY, M., «Les processus d'internationalisation du droit pénal (criminalité économique et atteintes à la dignité de la personne)», Archives de politique criminelle, n. 23 (2011), p. 123, citada por MICHEL, P., «La conformité constitutionnelle de l'intégration de la notion d'identité de genre dans le droit pénal», en HUTIER, S., MICHEL, P., CATELAN, N., PERRIER, J. B., Jurisprudence du Conseil Constitutionnel, n. 111, 2017/3, pp. 713-748, p. 722.

${ }^{33}$ Ver LAURENZO COPELLO, P., «¿Hacen falta figuras generoespecíficas para proteger mejor a las mujeres?», Estudios Penales $y$ Criminológicos, vol. XXXV (2015), pp. 796 a 798. Ver también supra, nota a pie de página n. 30.

${ }^{34}$ Informe explicativo del Convenio de Estambul, ob. cit., puntos 198 y 203, págs. 37 y 38.
} 
perspectiva de género en Derecho penal, haciendo primar dicho enfoque sobre las exigencias de neutralidad penal.

Como precisa Patsilí Toledo, «hasta ahora, la mayor parte de las legislaciones -tanto civiles como penales- ha abordado la violencia contra las mujeres a partir de dos grandes restricciones: (1) se limitan a la violencia que ocurre en la esfera doméstica o privada, y (2) la protección se otorga en términos neutros en cuanto a género, a fin de resguardar la igualdad formal. Así, la protección consecuente se enfoca, en el plano simbólico y normativo, no en las mujeres -aunque sean éstas las principales víctimas de la violencia doméstica, intrafamiliar o en la pareja- sino en ciertas relaciones o vínculos que se estiman merecedores de una protección especial por parte de la ley, pues son parte o pueden serlo, de relaciones de familia» ${ }^{35}$. El motivo por el que dichas excepciones en Derecho penal son poco frecuentes es la importancia que se atribuye a la neutralidad penal, exigencia que recae sobre la Ley para mantenerse indiferente ante las características de la víctima y el fuero interno del agresor, de cara a proteger la seguridad jurídica, de la que son garantes la abstracción e impersonalidad de la norma.

Es necesario sin embargo señalar el limitado alcance que el Convenio otorga a dicho enfoque, puesto que no precisa recomendaciones semejantes con respecto al resto de infracciones. También hubiese sido deseable que el texto sugiriese el uso de la perspectiva en cuestión para el análisis de otras disposiciones penales, como la legítima defensa en los casos de violencia de género en el ámbito sentimental ${ }^{36}$. Sea como fuere, del CE se espera que la introducción de la perspectiva de género cale también en el Derecho interno de los estados signatarios. Sin embargo, ciertas incoherencias y ambigüedades internas merman su potencial transformador. El CE ha supuesto sin duda novedades incuestionables, pero, tras seis años en vigor, conviene también señalar sus insuficiencias.

\footnotetext{
${ }^{35}$ Ver TOLEDO VÁSQUEZ, P., «La controversial tipificación del femicidio / feminicidio. Algunas consideraciones penales y de derechos humanos», Biblioteca virtual del Observatorio de Violencia Social y de Género de México, 2007, p. 10. Disponible a través del siguiente enlace: http://ovsyg.ujed.mx/documentos/ [15/10/2020].

${ }^{36} \mathrm{El} \mathrm{CE}$ tampoco precisa indicaciones de este tipo para mecanismos penales cuya ceguera al género ya había sido cuestionada. Ver, por ejemplo, para el caso de la legítima defensa en los casos de violencia de género en el ámbito sentimental, la proposición de Ley francesa para instaurar una presunción de legítima defensa para las violencias conyugales, del 11 de septiembre de 2019, disponible online: http://www.assemblee-nationale.fr/15/pdf/propositions/pion2234.pdf, planteada a raíz del caso Jacqueline Sauvage (https://www.lemonde.fr/idees/article/2016/01/11/creons-un-etat-de-legitime-defense-differee 4845003 3232.html, https://www.nouvelobs.com/justice/20160202.OBS3875/affaire-sauvage-vers-une-legitime-defense-differee-pour-femmes-battues.html). En situaciones como el caso Sauvage, puede suceder que las respuestas a las agresiones no se cometan con la inmediatez requerida ni cumplan con el criterio de defensa racional, es decir, con una defensa adecuada para impedir o repeler la agresión inicial ilegítima. Ver también el análisis de LE MAGUERESSE, C., «Les femmes victimes des violences de leur conjoint et la légitime défense», pp. 217- 232, en PICHARD, M. y VIENNOT, C., (dirs.), Le traitement juridique et judiciaire des violences conjugales, Mare et Martin, 2016. Dicho planteamiento ya había sido asumido por el Derecho canadiense mediante la figura de la «legítima defensa diferida». Ver el artículo 34, sección 2, f.1., del Código Penal Canadiense. Todos los enlaces mencionados han sido consultados el 15/10/2020.
} 
3. Las carencias: la vaguedad conceptual, un posible freno a la capacidad transformadora del texto

El Convenio introduce una serie de conceptos esenciales para la correcta adopción de medidas para la lucha contra la violencia contra las mujeres. El problema que se plantea en este caso es que los redactores, intentando mantener la coherencia a nivel interno, han optado por no definirlos, cediendo a las Partes la libertad de delimitarlos conceptualmente. Al no marcar directrices ni establecer una definición unívoca, esta ampliación competencial a favor de los Estados corre el riesgo de hacer de los conceptos elementos instrumentales y, en el peor de los casos, puede provocar el desarrollo de una regulación europea todavía más heterogénea de lo que ya es.

\subsection{La «perspectiva de género», una herramienta no definida}

El artículo 6 del CE aborda lo que él mismo llama «Políticas sensibles al género», líneas de actuación que implican la adopción de la perspectiva de género en el diseño y elaboración de cualquier medida que venga a implementar el texto internacional. Estas medidas pueden afectar a la materia legislativa penal para los casos que previamente hemos mencionado, pero también afectar a la elaboración de políticas públicas en materia de sensibilización. En cualquier caso, lo que parece evidente es que los redactores han hecho de este enfoque la piedra angular del desarrollo del texto, reconociendo implícitamente su potencial académico y científico, y sobrepasando una concepción meramente política de la perspectiva. El citado Informe explicativo del Convenio de Estambul ${ }^{37}$ señala que dicho artículo se aplica también al resto de disposiciones del CE, convirtiéndose en una suerte de vector-guía.

No obstante, como decíamos previamente, el CE omite explicitar lo que ha de entenderse por perspectiva de género. De su lectura se desprende que su finalidad es promover la autonomía de las mujeres mediante la potenciación de la igualdad real, por lo que entendemos que dicho enfoque se ha adoptado como objetivo y como herramienta metodológica.

En este sentido, siguiendo el análisis que Maggy Barrère hace del Gender mainstreaming ${ }^{38}$, creemos que sería útil detallar la finalidad «social» de la inclusión de dicha perspectiva, es decir, afinar

\footnotetext{
${ }^{37}$ Informe explicativo del Convenio de Estambul, ob. cit.

${ }^{38}$ BARRERE UNZUETA, M., «La interseccionalidad como desafío al mainstreaming de género en las politicas públicas», Revista Vasca de Administración Pública, núm. 87-88/2010., pp. 225-252. El texto completo está disponible a través del siguiente enlace: https://dialnet.unirioja.es/ejemplar/259680 [15/10/2020].
} 
su contenido en lo que «incorpora, integra o incluye en todas las esferas político-normativas» ${ }^{39}$. De otro modo, si carece de unidad, la perspectiva en cuestión corre el riesgo de convertirse en un «concepto puramente instrumental ${ }^{40}$. En aras de lograr esa autonomía convendría que se especificasen pautas para asumir jurídicamente esas desigualdades. En caso contrario, es probable que la perspectiva de género no consiga el alcance que el CE le otorga. Dicha falta de precisión se traduce en reformas ambiguas, como, por ejemplo, la operada en Francia en materia de violencia sexista. A raíz del Convenio, dicha reforma ha introducido la citada circunstancia agravante general por motivos sexistas, que viene fundamentalmente a sancionar las agresiones cometidas contra mujeres por el mero hecho de serlo, pero que, sin embargo, ha terminado por convertirse en un dispositivo ineficaz, incompatible con la protección de las violencias conyugales, y redactado en términos genderblind. La falta de claridad también tiene repercusiones en el texto mismo del CE, por ejemplo, en la opacidad con la que define el concepto de violencia doméstica, un problema que reconoce ligado fundamentalmente a mujeres, pero que entiende en términos neutros.

\subsection{La opacidad del concepto de violencia doméstica}

Otro de los reproches que se le han hecho justificadamente al texto del CE consiste en la ambigüedad con la que se acota la violencia doméstica, dado que no queda claro si es una forma de violencia contra las mujeres, de violencia de género, o bien un fenómeno independiente ${ }^{41}$. Ya el mismo título del Convenio («Convenio del Consejo de Europa sobre prevención y lucha contra la violencia contra la mujer y la violencia doméstica») parece considerar incluir la violencia doméstica y la violencia contra las mujeres como dos categorías distintas pero anejas, «cuando habitualmente [en el ámbito internacional] se ha entendido la primera como una forma específica de la segunda» ${ }^{42}$.

Más adelante, el artículo 2 señala que «el Convenio se aplicará a todas las formas de violencia contra las mujeres, incluida la violencia doméstica, que afecta a las mujeres de manera desproporcionada». Sin embargo, el Preámbulo viene, en cierta medida, a matizar lo anterior, «reconociendo que [...] los hombres también pueden ser víctimas». Siguiendo esta lógica genderblind,

\footnotetext{
${ }^{39}$ Ibid, p. 243.

${ }^{40} \mathrm{Id}$.

${ }^{41}$ Para entender los motivos por los que los redactores del CE adoptaron el sorprendente enfoque en cuestión, conviene hacer una lectura del análisis que Truchero y Arnáiz realizan sobre las discrepancias que surgieron entre varios Estados acerca de este tema durante la fase de elaboración del texto. Ver TRUCHERO, J.; ARNÁIZ, A., «Aproximación al Convenio europeo de violencia contra las mujeres y la violencia doméstica», Revista Europea de Derechos Fundamentales, n. 19/1er Semestre 2012, pp. 123-156, p. 142.

${ }^{42} I b i d$., p. 141. Ver también VENTURA FRANCH, A., ob. cit., p. 201 y ss.
} 
el artículo 3 b. del CE presenta la violencia doméstica -de la que la conyugal forma parte- en términos neutros ${ }^{43}$, introduciendo un «enfoque cuyo paradigma no es el desequilibrio de poder en la sociedad sino la especial relación de intimidad en la familia» ${ }^{44}$.

Conviene analizar todo lo anterior bajo la óptica que precisa Magali Thill, en la medida en que entiende que el «término "doméstica", que remite al lugar donde se produce la violencia, invisibiliza en efecto una violencia cuyo origen es la jerarquía sexual y refuerza la dicotomía público/privado que, desde su consagración en la teoría del contrato social/sexual, ha apuntalado la desigualdad estructural entre mujeres y hombres» ${ }^{45}$. Como precisa Marc Pichard «la toma en consideración contemporánea de las violencias conyugales es principalmente el resultado de instrumentos jurídicos que tienen como objetivo -no siempre exclusivo- la protección de los derechos de las mujeres. Dicho en otros términos, mientras que los textos que se producen son genderblind y pueden por tanto aplicarse a sujetos que no sean mujeres, es la figura de la mujer víctima de violencia la que anima la producción jurídica, tanto en Francia como en el extranjero» ${ }^{46}$.

\subsection{La trata con fines de explotación sexual, una violencia olvidada}

La insuficiencia del Tratado también se hace patente en lo relativo a la trata con fines de explotación sexual, violencia que finalmente quedó excluida del ámbito de aplicación del CE porque existían otros mecanismos internacionales específicamente pensados para abordarla. El punto 154 del citado Informe de evaluación de referencia recuerda la intención de los redactores de «evitar cubrir los mismos comportamientos que otros Convenios del Consejo de Europa ya cubrían, en concreto, el Convenio sobre la lucha contra la trata de seres humanos (STCE $\left.n^{\circ} 197\right)$ y el Convenio sobre la protección de la infancia contra la explotación y el abuso sexual (STCE nº 201)». Simbólicamente, hubiese sido más coherente que un texto de este calado -primer convenio vinculante a nivel regional europeo en la materiamencionara este tipo de violencia, en particular, porque dicha omisión conlleva ciertas repercusiones prácticas en la actividad del GREVIO. Por ejemplo, en el Informe francés en cuestión, el GREVIO

\footnotetext{
${ }^{43}$ Art. 3.b. del CE: «se entenderán todos los actos de violencia física, sexual, psicológica o económica que se producen en la familia o en el hogar o entre cónyuges o parejas de hecho antiguos o actuales, independientemente de que el autor del delito comparta o haya compartido el mismo domicilio que la víctima».

${ }^{44}$ Ver TRUCHERO, J., ARNÁIZ, A., ob. cit., pág. 141.

${ }^{45}$ THILL, M., «El Convenio de Estambul: Un análisis crítico y contextualizado», Labrys, Études féministes/Estudios feministas, julio 2017junio 2018, p. 2, disponible online: https://www.labrys.net.br/labrys31/criminologie/magali.htm [15/10/2020].

${ }^{46}$ Ver PICHARD, M., «Les violences conjugales comme violences faites aux femmes», pp. 15 - 25, en PICHARD, M., y VIENNOT, C., (dirs.), Le traitement juridique et judiciaire des violences conjugales, Mare et Martin, 2016, p. 17. La traducción es obra de la autora.
} 
descarta estudiar este ámbito del problema, precisamente porque el campo de aplicación del CE no lo incluye. A la luz del punto 12 consta que «el convenio de Estambul no cubre la prostitución [forzada] como tal. [...]» [Por tanto], «No pertenece al mandato del GREVIO examinar esta dimensión» ${ }^{47}$.

La Plataforma Estambul Sombra, a la que se han adherido alrededor de 250 organizaciones autonómicas y nacionales, lo denunciaba recientemente en un Informe publicado en 2018: «Aunque contamos con el Convenio sobre la lucha contra la trata de personas y con el Grupo de Expertos (GRETA) del Consejo de Europa, sería útil que el GREVIO la visibilice [la trata con fines de explotación sexual] como violencia de género» ${ }^{48}$, conforme a la Resolución del Parlamento Europeo de 26 de febrero de 2014, sobre explotación sexual y prostitución y su impacto en la igualdad de género. Ello probablemente conllevaría una mejor aplicación de la Ley 12/2009, de 30 de octubre, reguladora del derecho de asilo y de la protección subsidiaria. Sobre dicha Ley, cuyo texto reconoce en su artículo 6 a) que las violencias sexuales pueden ser consideradas como un motivo de persecución, Inmaculada Montalbán apunta que «existen datos que revelan una aplicación restringida» ${ }^{49}$. Así, la interpretación de su texto a la luz del CE contribuiría a reforzar el reconocimiento de las violencias sexuales que padecen las mujeres que se ven atrapadas en las redes de trata sexual como violencias ejercidas contra las mujeres por motivos de género. Conforme al artículo 60 del CE y el punto 311 del Informe explicativo citado ${ }^{50}$, la existencia de dichas violencias justificaría la concesión del asilo político ${ }^{51}$.

\subsection{El consentimiento sexual: un concepto central indeterminado}

Como hemos visto, en el texto del CE existe una serie de expresiones cuyo contenido no define. Se diría que quienes lo redactaron quisieron hacer de varias de ellas elementos fundamentales de la lucha contra la violencia ejercida contra las mujeres, pero, sorprendentemente, prefirieron ceder a los Estados

\footnotetext{
${ }^{47}$ Informe de evaluación de referencia (Francia), ob. cit., punto 5, p.12.

${ }^{48}$ Plataforma Estambul Sombra, Informe Sombra al GREVIO de 2018, p. 25.

${ }^{49}$ Ver MONTALBÁS HUERTAS, I., Trata sexual de mujeres: nuevas claves para el derecho de asilo, C.G.P.J., Escuela Judicial, Estudios, p. 4, recurso online.

${ }^{50}$ En efecto, el artículo 60 del CE y el punto 311 del Informe explicativo del Convenio de Estambul, ob. cit., estiman pertinente recordar que sobre las Partes signatarias recae la «obligación de adoptar las medidas necesarias para conseguir que la violencia basada en el género pueda llegar a ser reconocida como una forma de persecución en el sentido del artículo 1 A (2) [de la Convención de Ginebra de 1951 sobre el estatuto de los refugiados]» $\mathrm{y}$ 《conducir por tanto a que se le otorgue el estatuto de refugiada» a las mujeres que se encuentren en tales situaciones.

${ }^{51}$ MONTALBÁS HUERTAS, I., ob. cit., pág. 20. Ver también BLÁZQUEZ VILLAPLANA, B., «La trata de personas con fines de explotación sexual en España: elementos para la reflexión», Revista Espiga, 16, n. ${ }^{\circ} 34$ (julio-diciembre, 2017), pp. 183-196, disponible a través del siguiente enlace: https://dialnet.unirioja.es/servlet/articulo?codigo=6358676 [15/10/2020], o, por fin, THILL, M., y GIMÉNEZ ARMENTIA, P., «El enfoque de Género: un requisito necesario para el abordaje de la trata de seres humanos con fines de explotación sexual», Revista Europea de Derechos Fundamentales, primer semestre 2016: 27, pp. 439-459.
} 
la tarea de darles contenido. En este apartado nos centraremos en la noción de ausencia de consentimiento para los casos de violencia sexual, concepto del que debería derivarse cierta mejora en la materia, en la medida en que supone redefinir los contornos penales de las agresiones sexuales, las cuales, a priori, pasarían a ser más garantistas para las víctimas ${ }^{52}$.

Catherine Le Magueresse entiende que la configuración del delito de violación parte de la presunción de un consentimiento otorgado ${ }^{53}$. Para que exista delito, dicho consentimiento debe ser anulado, y, por tanto, previamente, debe haber existido ${ }^{54}$. El planteamiento del «solo sí es sí» viene a reestructurar la configuración de los delitos sexuales, de manera que, en lugar de tener que probar la existencia de alguna violencia o intimidación que hubiera anulado el consentimiento, habrá que establecer, en primer lugar, la existencia misma de ese consentimiento. Esta lógica estructurante podría tener cabida, de alguna manera, en el texto del CE, concretamente, a través de su artículo 36, que establece que «el consentimiento debe prestarse voluntariamente como manifestación del libre arbitrio de la persona considerado en el contexto de las condiciones circundantes». El punto 193 del citado Informe explicativo considera que «en la puesta en marcha de esta disposición, las partes de la convención están obligadas a adoptar una legislación penal que integre la noción de ausencia de libre consentimiento a los diferentes actos sexuales enumerados en los puntos a) a c)»> 55 .

Hasta ahora, la mayoría de las regulaciones penales europeas en materia de delitos sexuales se ha construido en torno a la apreciación de la ausencia de consentimiento condicionada a las características del comportamiento del agresor. Es decir, que la falta de voluntad de la víctima se aprecia a la luz de la violencia física o psicológica que hubiese podido padecer por parte del agresor. El texto del CE viene a reconsiderar este enfoque desde una perspectiva positiva del consentimiento, haciendo que solo la

\footnotetext{
${ }^{52}$ En España, el debate ha calado hondo a raíz del reciente caso conocido como La Manada, a través del que se ha vuelto a plantear la reforma de los delitos sexuales bajo el planteamiento «solo sí es sí». Ver la Sentencia del Tribunal Supremo, Sala de lo Penal, n. $344 / 2019$. ${ }^{53}$ Ver LE MAGUERESSE, C., «Viol et consentement en droit pénal français. Réflexions à partir du droit pénal canadien», Archives de politique criminelle, 2012/1 ( $\left.\mathrm{n}^{\circ} 34\right)$, pp. 223-240, disponible online: https://www.cairn.info/revue-archives-de-politique-criminelle-20121-page-223.htm [15/10/2020].

${ }^{54}$ Para que haya agresión sexual en el Derecho penal español, es necesaria la existencia de violencia o intimidación. Cualquiera de estos dos elementos anula el consentimiento de la víctima, forzada a cometer el acto sexual. Por ende, en la medida en que el comportamiento del agresor anula el consentimiento, se entiende que, previamente, ese consentimiento sí existía. Según el análisis de Le Magueresse, Id., la configuración de los delitos sexuales se basaría en una supuesta presunción (en sentido amplio) de un consentimiento implícito, establecido y normativo, anterior a la violencia o a la intimidación, y que sería síntoma de una visión androcéntrica de la realidad, en la medida en que entendería que las mujeres, por norma general, estarían dispuestas al acto sexual.

${ }^{55}$ Informe explicativo del Convenio de Estambul, CETS n. 2010, Consejo de Europa, 2011, punto 193, p. 36. Ver también el punto 192. Los actos en cuestión son los siguientes: «la penetración vaginal, anal u oral no consentida, con carácter sexual, del cuerpo de otra persona con cualquier parte del cuerpo o con un objeto, a los demás actos de carácter sexual no consentidos sobre otra persona», y, por fin, el «hecho de obligar a otra persona a prestarse a actos de carácter sexual no consentidos con un tercero».
} 
voluntad libre y manifiesta avale la validez del consentimiento dado ${ }^{56}$, desplazando el foco de atención del comportamiento del agresor, es decir, si hubo o no violencia, para ponerlo directamente en la víctima, esto es, si la víctima accedió o no al acto sexual, independientemente del comportamiento del agresor, otorgando así al concepto de consentimiento un valor central en la definición del delito ${ }^{57}$.

Sin embargo, lo cierto es que, a pesar de que mediante la ratificación del Convenio los Estados se comprometen a modificar sus legislaciones, integrando el concepto en cuestión, actualmente, pocos son los que ya han dado el paso $^{58}$. El Informe de evaluación que el GREVIO publicó el pasado noviembre de 2019 acerca del país galo denunciaba la situación francesa. «[La] redacción actual adoptada por el legislador francés pone el acento sobre los elementos probatorios que permiten constatar la ausencia de consentimiento en detrimento de la centralidad de la ausencia de consentimiento. Alineándose sobre las preconizaciones del Convenio, una definición de las violencias sexuales centrada sobre la ausencia de un consentimiento libre permitiría, según el GREVIO, paliar las insuficiencias que emergen de la situación actual. [...] Una definición así permitiría sobre todo operar el cambio de paradigma necesario para reconocer la centralidad que recae sobre la voluntad de la víctima, consiguiendo que Francia se sume a los países que ya han dado este paso importante» ${ }^{59}$.

A la luz de las lecturas citadas, y dada la centralidad y el valor que se otorga al consentimiento, sorprende que, sin embargo, «los redactores ha[yan] cedido a las Partes la tarea de decidir la formulación exacta de la legislación y los factores considerados como exclusivos de un consentimiento libre» ${ }^{60}$. De nuevo, si el Tratado no establece directrices más específicas, este planteamiento corre el riesgo de suponer más trabas que beneficios, ya sea, por ejemplo, de cara a la labor legislativa, que dependerá de los vaivenes políticos, ya sea en la labor jurisprudencial.

\footnotetext{
${ }^{56}$ LE MAGUERESSE, C., «Viol et consentement en droit pénal français», ob. cit. Ver también FRAISSE, G., Du consentement, París, Éd. Du Seuil, 2da edición, 2017, p. 7: El acto de consentir reviste un carácter político que encarna la libertad de elegir, así como «la capacidad de decir no a un orden injusto»».

${ }^{57}$ Ver, en este sentido, el punto 191 del Informe explicativo del Convenio de Estambul, en el que cita la sentencia Mc C/ Bulgaria de 4 de diciembre de 2003 de la Corte Europea de Derechos Humanos.

${ }^{58}$ En España, el actual Anteproyecto de Ley Orgánica de garantía integral de la libertad sexual, cuya tramitación fue aprobada el pasado 3 de marzo de 2020 por el Consejo de Ministros, pretende modificar la configuración de los delitos sexuales para incluir el planteamiento del «solo sí es sí». Ver también supra, nota a pie de página n. 52.

${ }^{59}$ Informe de evaluación de referencia (Francia), ob. cit., punto 192, p. 61.

${ }^{60}$ Informe explicativo del Convenio de Estambul, ob. cit., punto 193.
} 


\section{Conclusión}

El Convenio de Estambul supone, sin duda, un gran paso para la lucha contra la violencia de carácter machista. Al ser vinculante, se espera que los Estados que lo han ratificado adopten las medidas necesarias para implementarlo, introduciendo en Derecho interno principios de soft law. Ello implicará modular una serie de conceptos cuya asunción, frecuente en Derecho internacional debido a su mayor permeabilidad a los derechos humanos, no lo era tanto en las legislaciones nacionales. Entre dichos conceptos figura el relativo a la «violencia ejercida contra las mujeres por motivos de género», el cual, dada la amplitud con la que lo define el texto del CE, debería suponer que dicha violencia fuera reconocida en todas sus vertientes por los distintos Estados. Por otro lado, merece la pena hacer hincapié en la generoespecificidad que podría caracterizar la configuración de ciertos delitos. Se trata de un tema que crea grandes controversias y que cuenta con escaso apoyo. De hecho, a nivel europeo, España es quizá el único país que dispone de un dispositivo generoespecífico para sancionar la violencia de género. Si los países signatarios son fieles al texto, nuestro sistema jurídico dejaría de ser una excepción en Europa.

En todo caso, como hemos visto, pasado el entusiasmo inicial, el balance de los logros llevados a cabo por el CE deja la amarga sensación de un proyecto inacabado, debido en parte a conceptos indeterminados que se presentan sin embargo como centro neurálgico de medidas progresistas y transformadoras. En definitiva, se trata de un proyecto al que deben serle reconocidos grandes méritos, pero con respecto al cual no hay que olvidar que las reformas internas necesitan un impulso que, para bien o para mal, debe provenir de las fuerzas políticas.

\section{Coda}

Si como dice H. Arendt, a diferencia de la culpa, la responsabilidad se puede entender como la asunción colectiva de una deuda en cierto sentido ineludible y que puede pasar de una generación a otra, la lucha contra la violencia ejercida sobre la mujer sigue siendo algo que incumbe, se quiera o no, a todos. Algunos, conscientes de su trascendencia, hicieron bandera de ello. Es el caso del profesor M. Calvo García al que está dedicado el presente trabajo. 


\section{Referencias bibliográficas}

AGUILAR CÁRCELES, M. M. «Circunstancias agravantes genéricas», en MORILLAS CUEVA (dir.), Estudios sobre el Código Penal reformado, Madrid, 2015, pp. 58-63.

ALIJA FERNÁNDEZ, R., «La violencia doméstica contra las mujeres y el desarrollo de estándares normativos de derechos humanos en el marco del Consejo de Europa», Revista General de Derecho Europeo, 24, 2011.

BROX SÁENZ DE LA CALZADA, A., «Una aproximación al concepto de género en Derecho Penal francés y español. De la polémica a su validez constitucional», Cuadernos Electrónicos de Filosofía del Derecho, n. 40, junio 2019, pp. 23-44.

BARRERE UNZUETA, M., «La interseccionalidad como desafío al mainstreaming de género en las políticas públicas», Revista Vasca de Administración Pública, n. 87-88, 2010, pp. 225-252.

BLÁZQUEZ VILLAPLANA, B., «La trata de personas con fines de explotación sexual en España: elementos para la reflexión», Revista Espiga, 16, n. 34 (julio-diciembre, 2017), pp. 183-196.

CALVO GARCÍA, M., «La violencia de género como violación de Derechos Humanos. El papel de los movimientos sociales en la lucha por los derechos», en A.A.V.V., Historia de los Derechos Fundamentales, Tomo IV, Siglo XX, Madrid, Dykinson, 2014.

DELMAS-MARTY, M., «Les processus d'internationalisation du droit pénal (criminalité économique et atteintes à la dignité de la personne)», Archives de politique criminelle, n. 23, 2011.

DÍEZ RIPOLLÉS, J. L.; CEREZO DOMÍNGUEZ, A. I.; BENÍTEZ JIMÉNEZ, Ma . J., La política criminal contra la violencia sobre la mujer pareja (2004-2014). Su efectividad, eficacia y eficiencia, Valencia, Tirant lo Blanch, 2018.

DIONISI-PEYRUSSE, A.; PICHARD, M., «La prise en compte des violences conjugales en matière d'autorité parentale», Actualité juridique. Famille, Dalloz, 2018, pp. 34-37.

EDWARDS, A., Violence against women under International Human Rights Law, Cambridge University Press, 2011.

FRAISSE, G., Du consentement, Éd. Du Seuil, 2da edición, 2017, 160 págs.

FRIEDMAN, E., J, «Women's human rights: the emergence of a movement perspective», en PETERS, J., y WOLPER, A., (eds.), Women's Rights, Human Rights: International Feminist Perspectives, Nueva York, Routledge, 1995.

GARCÍA INDA, A., y LOMBRADO, E., (coords.), Género y Derechos Humanos, Terceras Jornadas Derecho Humanos y libertades fundamentales, Huesca, Mira Ed., 2002.

GIL RUIZ, J. M. (ed.), El Convenio de Estambul como marco de derecho antisubordiscriminatorio, Madrid, Dykinson S.L., 2018.

LAURENZO COPELLO, P., «La violencia de género en el derecho penal: Un ejemplo de paternalismo punitivo», en Género, violencia y Derecho, LAURENZO, P., MAQUEDA, Mª. L, RUBIO, A., (coords.) Valencia, Tirant lo blanch, 2008, pp. 329361.

LAURENZO COPELLO, P., «¿Hacen falta figuras generoespecíficas para proteger mejor a las mujeres?», Estudios Penales y Criminológicos, vol. XXXV, 2015, pp. 783-830.

LE MAGUERESSE, C., «Les femmes victimes des violences de leur conjoint et la légitime défense», pp. 217-232, en PICHARD, M. y VIENNOT, C. (dirs.), Le traitement juridique et judiciaire des violences conjugales, Mare et Martin, 2016.

LE MAGUERESSE, C., «Viol et consentement en droit pénal français. Réflexions à partir du droit pénal canadien», Archives de politique criminelle, 2012/1, n. 34, pp. 223-240.

LOUSADA AROCHENA, J. F., «El convenio del Consejo de Europa sobre prevención y lucha contra la violencia contra las mujeres y la violencia de género», AequAlitaS, 2014, n. 35, pp. 6-15. 
MAQUEDA ABREU, Ma . L., «¿Es la estrategia penal una solución a la violencia contra las mujeres?», InDret, 2007, n. 407.

MARÍN DE ESPINOSA CEBALLOS, E., «La agravante genérica de discriminación por razones de género (art. 22.4 CP)», Revista Electrónica de Ciencia Penal y Criminología, RECPC, 2018, n. 20-27, pp. 1-20.

MICHEL, P., «La conformité constitutionnelle de l'intégration de la notion d'identité de genre dans le droit pénal», en HUTIER, S., MICHEL, P., CATELAN, N., PERRIER, J. B., Jurisprudence du Conseil Constitutionnel, n. 111, 2017/3, pp. 713-748.

MONTALBÁN HUERTAS, I., Trata sexual de mujeres: nuevas claves para el derecho de asilo, C.G.P.J., Escuela Judicial, Estudios, material docente online.

PICHARD, M., «Les violences conjugales comme violences faites aux femmes», pp. 15-25, en PICHARD, M., y VIENNOT, C. (dirs.), Le traitement juridique et judiciaire des violences conjugales, Mare et Martin, 2016.

RUEDA MARTÍN, Ma . A., "Cometer un delito por discriminación referente al sexo de la víctima y/o por razones de género como circunstancia agravante genérica», Revista Electrónica de Ciencia Penal y Criminología, RECPC 21-04 (2019), pp. 137.

SEOANE MARÍN, Mª . J., OLAIZOLA NOGALES, I., «Análisis de la circunstancia agravante de discriminación por razones de género (22.4 CP)», Estudios Penales y Criminológicos, vol. XXXIX (2019).

SMART, C., «La teoría feminista y el discurso jurídico», en BIRGIN, H. (comp.), El derecho en el género y el género en el derecho, Buenos Aires, Biblios, 2000, pp. 31-71.

THILL, M., «El Convenio de Estambul: Un análisis crítico y contextualizado», Labrys, Études féministes/Estudios feministas, n. 31, julio 2017- junio 2018.

THILL, M., y GIMÉNEZ ARMENTIA, P., «El enfoque de Género: un requisito necesario para el abordaje de la trata de seres humanos con fines de explotación sexual», Revista Europea de Derechos Fundamentales, primer semestre 2016: 27, pp. 439459 .

TOLEDO VÁSQUEZ, P., «La controversial tipificación del femicidio / feminicidio. Algunas consideraciones penales y de derechos humanos», Biblioteca virtual del Observatorio de Violencia Social y de Género de México, 2007.

TRUCHERO DÍAZ, J., ARNÁIZ, A., «Aproximación al Convenio europeo de violencia contra las mujeres y la violencia doméstica», Revista Europea de Derechos Fundamentales, n. 19/1er Semestre 2012, pp. 123-156.

VENTURA FRANCH, A., «El CE y los sujetos de la violencia de género. El cuestionamiento de la violencia doméstica como categoría jurídica», UNED. Revista de Derecho Político, n. 97, 2016.

\section{Fuentes documentales}

Consejo de Europa, Informe explicativo del Convenio de Estambul, Rapport explicatif de la Convention du Conseil de l'Europe sur la prévention et la lutte contre la violence à l'égard des femmes et la violence domestique, CETS n. 2010, 2011, versión francesa.

Delegación para las Víctimas, Ministerio del Interior Francés, Informe Principales datos sobre la Encuesta sobre las muertes violentas en el ámbito de la pareja, año 2016.

Delegación para las Víctimas, Ministerio del Interior Francés, Estudio nacional sobre las muertes violentas en el ámbito de la pareja, año 2017.

Delegación para las Víctimas, Ministerio del Interior Francés, Estudio nacional sobre las muertes violentas en el ámbito de la pareja, año 2018. 
Grupo GREVIO, Rapport sur les mesures d'ordre législatif et autres donnant effet aux dispositions de la Convention du Conseil de l'Europe sur la prévention et la lutte contre la violence à l'égard des femmes et la violence domestique (France), GREVIO/Inf(2019)16, Consejo de Europa, Francia, noviembre de 2019.

Grupo GREVIO, Grevio Baseline Evaluation Report on legislative and other mesures giving effect to the provisions of the Council of Europe Convention on Preventing and Combating Violence against Women and Domestic Violence (Spain), GREVIO/Inf(2020)19, Consejo de Europa, Francia, noviembre de 2020.

Plataforma Sombra Estambul España, Informe Sombra al GREVIO 2018. 